\title{
Integrated-boost IMRT or 3-D-CRT using FET-PET based auto-contoured target volume delineation for glioblastoma multiforme - a dosimetric comparison
}

\author{
Marc D Piroth*1,4, Michael Pinkawa1,4, Richard Holy¹,4, Gabriele Stoffels ${ }^{3,4}$, \\ Cengiz Demirel ${ }^{1}$, Charbel Attieh${ }^{1}$, Hans J Kaiser ${ }^{2}$, Karl J Langen ${ }^{3,4}$ and \\ Michael J Eble $e^{1,4}$
}

Address: ${ }^{1}$ Department of Radiation Oncology, RWTH Aachen University Hospital, Pauwelsstrasse 30, 52074 Aachen Germany, ${ }^{2}$ Department of Nuclear Medicine, RWTH Aachen University Hospital, Pauwelsstrasse 30, 52074 Aachen Germany, ${ }^{3}$ Institute of Neurosciences and Medicine, Research Centre Jülich, 52425 Jülich, Germany and ${ }^{4}$ JARA (Jülich Aachen Research Alliance) Forschungszentrum Jülich GmbH Wilhelm-JohnenStraße, 52428 Jülich, Germany

Email: Marc D Piroth* - mpiroth@ukaachen.de; Michael Pinkawa - mpinkawa@ukaachen.de; Richard Holy - rholy@ukaachen.de; Gabriele Stoffels - g.stoffels@fz-juelich.de; Cengiz Demirel - cdemirel@ukaachen.de; Charbel Attieh - cattieh@ukaachen.de; Hans J Kaiser - hjkaiser@ukaachen.de; Karl J Langen - k.j.langen@fz-juelich.de; Michael J Eble - meble@ukaachen.de

* Corresponding author

Published: 23 November 2009

Radiation Oncology 2009, 4:57 doi:10.1186/1748-717X-4-57

This article is available from: http://www.ro-journal.com/content/4/I/57

(C) 2009 Piroth et al; licensee BioMed Central Ltd.

This is an Open Access article distributed under the terms of the Creative Commons Attribution License (http://creativecommons.org/licenses/by/2.0), which permits unrestricted use, distribution, and reproduction in any medium, provided the original work is properly cited.
Received: 13 August 2009

Accepted: 23 November 2009

\begin{abstract}
Background: Biological brain tumor imaging using O-(2-[ $\left.{ }^{18} \mathrm{~F}\right]$ fluoroethyl)-L-tyrosine (FET)-PET combined with inverse treatment planning for locally restricted dose escalation in patients with glioblastoma multiforme seems to be a promising approach.

The aim of this study was to compare inverse with forward treatment planning for an integrated boost dose application in patients suffering from a glioblastoma multiforme, while biological target volumes are based on FET-PET and MRI data sets.

Methods: In 16 glioblastoma patients an intensity-modulated radiotherapy technique comprising an integrated boost (IB-IMRT) and a 3-dimensional conventional radiotherapy (3D-CRT) technique were generated for dosimetric comparison. FET-PET, MRI and treatment planning CT (P-CT) were co-registrated. The integrated boost volume (PTVI) was auto-contoured using a cut-off tumor-tobrain ratio (TBR) of $\geq 1.6$ from FET-PET. PTV2 delineation was MRI-based. The total dose was prescribed to 72 and $60 \mathrm{~Gy}$ for PTVI and PTV2, using daily fractions of 2.4 and 2 Gy.

Results: After auto-contouring of PTVI a marked target shape complexity had an impact on the dosimetric outcome. Patients with 3-4 PTVI subvolumes vs. a single volume revealed a significant decrease in mean dose $(67.7$ vs. $70.6 \mathrm{~Gy})$. From convex to complex shaped PTVI mean doses decreased from 7I.3 Gy to 67.7 Gy. The homogeneity and conformity for PTVI and PTV2 was significantly improved with IB-IMRT. With the use of IB-IMRT the minimum dose within PTVI (6I.I vs. $57.4 \mathrm{~Gy}$ ) and PTV2 (5I.4 vs. $40.9 \mathrm{~Gy}$ ) increased significantly, and the mean EUD for PTV2 was improved (59.9 vs. $55.3 \mathrm{~Gy}, \mathrm{P}<0.0 \mathrm{I})$. The EUD for PTVI was only slightly improved (68.3 vs. 67.3 Gy). The EUD for the brain was equal with both planning techniques.
\end{abstract}


Conclusion: In the presented planning study the integrated boost concept based on inversely planned IB-IMRT is feasible. The FET-PET-based automatically contoured PTVI can lead to very complex geometric configurations, limiting the achievable mean dose in the boost volume. With IBIMRT a better homogeneity and conformity, compared to 3D-CRT, could be achieved.

\section{Introduction}

In spite of intensive efforts to improve treatment strategies the prognosis of patients suffering from a Glioblastoma multiforme remains poor with a median survival time of 12-14 months [1,2]. Even though a radiation doseresponse relationship could be demonstrated in clinical $[3,4]$ as well as in experimental studies [5-8], no significant increase of survival could be achieved in randomized clinical trials. Although several Phase II-studies showed promising results $[9,10]$, the RTOG 93-05 study failed to demonstrate a prognostic improvement for patients treated with a stereotactic boost in addition to the standard 60 Gy fractionated conformal radiotherapy with the alkylating agent carmustine (BCNU) [11]. The authors speculated that these results may be caused by the fact that glioblastomas (GBM's) are inherently infiltrating neoplasms. Another reason for the poor results of those studies, however, may be the inability of current imaging methods to adequately reflect the true extent of the tumors. Magnetic resonance imaging (MRI) is currently the method of choice for the diagnosis of primary brain tumors. The delineation between glioma and surrounding edema with MRI is unreliable since the tumor is not sharply demarcated and if in addition the blood-brain barrier remains intact. Therefore, it appears essential to base locally focused dose escalation concepts on more specific imaging methods, such as MR spectroscopy or Positron Emission Tomography (PET). Several data suggest that brain tumor imaging with PET using amino acids is more reliable than MRI to define the extent of cerebral gliomas [12-16]. O-(2-[18F]fluoroethyl)-L-tyrosine (FET) is a well established amino acid tracer for PET. Biological brain tumor imaging combined with inverse treatment planning for locally restricted dose escalation in patients with glioblastoma multiforme seems to be a promising approach.

The aim of this study was to compare inverse with forward treatment planning for an integrated boost dose application in patients suffering from a glioblastoma multiforme, while the auto-contoured biological target volumes are based on O-(2-[18F]Fluorethyl)-L-Tyrosin (FET)-PET and MRI data sets.

\section{Materials and methods Patients}

Sixteen consecutive patients with a histologically proven supratentorial glioblastoma multiforme (WHO grade IV) were treated with an intensity-modulated radiotherapy comprising an integrated boost dose application (IBIMRT). In addition a 3-dimensional conventional radiotherapy (3D-CRT) treatment plan was generated for dosimetric comparison. The selected patients were treated in our clinic from January 2008 to January 2009 within an ongoing prospective monocentric phase-II study. The mean age was 55.6 (36-73) years. Ten patients were male. The Karnofsky perfomance index was $\geq 70 \%$ in 15 patients. A gross total and partial resection could be achieved in 8 patients. The tumor was located in the right and left hemisphere in 4 and 12 patients. Half of the tumors were located in the frontal lobe, while the other half of patients showed an equally frequent location within the temporal or parietal lobe. The study was approved by the university ethics committee and federal authorities. All subjects gave written informed consent for their participation in the study.

\section{Target volume definition}

After head fixation with a thermoplastic mask (Orfit ${ }^{\circledR}$ Raycast $^{\oplus}$-HP mask system, mean target isocenter translation $<2 \mathrm{~mm}$ [17]) a dedicated computer tomography (P-CT) with continuous slices of $2 \mathrm{~mm}$ thickness was made. An O-(2-F-18-Fluorethyl)-L-Tyrosin-PET (FET-PET) was performed in all 16 patients within 2 days after P-CT and within an interval of 11-20 days after surgical resection or biopsy of the tumor. Prior to FET-PET patients remained fasting for at least $6 \mathrm{~h}$. PET images were acquired 15-40 min after intravenous injection of $200 \mathrm{MBq}{ }^{18} \mathrm{~F}-\mathrm{FET}$. The measurements were performed with an ECAT EXACT HR+ scanner (Siemens Medical Systems, Inc.) in 3-dimensional mode (32 rings; axial field of view, $15.5 \mathrm{~cm}$ ) (details s [18]).

All patients received pre- and postoperative MRI's, performed in a 1,5 tesla MRI scanner with a standard head coil, which were integrated in the planning process. The MRI protocol consisted of a contrast enhanced T1weighted, a T2-weighted and a FLAIR (fluid attenuation inversion recovery) sequence. All image data sets were reconstructed and imported into the Philips Pinnacle ${ }^{3}$ irradiation treatment planning system (Version $8.0 \mathrm{~m}$, Philips Medical Systems, Eindhoven, NL). The Philips Syntegra $^{\mathrm{TM}}$ image registration tool was used to co-registrate the postoperative MRI and FET-PET to the native P-CT. From the three auto-registration methods available in Syntegra the Mutual Information (MI) method was used 
[19]. The image co-registration process was performed automatically. Finally the fusion results were assessed visually based on anatomic landmarks. The preoperative MRI was integrated side-by-side in the planning process.

Two clinical target volumes (CTV) were generated. For delineation of CTV1, defined as biological target volume from postoperative FET-PET imaging, an auto-contouring process was used.

The definition of the biological target volume with PET is a critical issue. Due to the limited spatial resolution of 5 $\mathrm{mm}$ it is not possible to define the exact tumor border on PET images. Tumor delineation based on the mean background activity such as the tumor/brain ratio appears to be an adequate approach for the problem of tumor definition in amino acid studies [20]. In a previous biopsy controlled study we found for tumor tissue a mean lesionto-brain ratio of FET uptake of $2.6 \pm 0.9$ and $1.2 \pm 0.4$ for peritumoral tissue [15]. Others reported that best differentiation of tumor and non-tumoral tissue could be observed at tumor/brain ratios of 2.0 and $2.2[16,21]$. In the present study CTV1 was defined as the volume within a cut-off tumor-to-brain ratio (TBR) of $\geq 1$.6. Since this threshold value is in the lower range the tumor volume is overestimated and is assumed to contain a safety margin of approx. $5 \mathrm{~mm}$. Therefore no additional margin was given to between CTV1 and PTV1 (CTV1 = PTV1).

For generating the TBR a polygonal reference region was drawn over several axial P-CT slices, comprising a volume of $40-70 \mathrm{~cm}^{3}$ from the contra-lateral cerebral hemisphere. Then the mean activity value of the normal brain reference area was multiplied by the cut-off value for automatic delineation of CTV1. Finally manual corrections were done, since the activity in blood vessels or postoperative extracerebral soft tissue could be above the cut-off value [22]. Venous structures, visible in the co-registrated MRI, were excluded. CTV-subvolumes comprising less than 3 voxels (FET-PET voxel size $2 \times 2 \times 2.4 \mathrm{~mm}$ ) were deleted. We classified the shape of the target volume CTV1 into three categories: convex, concave and complex. The term "complex" describes a finger-shaped or cuttlefish like appearance. In addition the number of separate subvolumes for each CTV1 was considered for classification of target volume complexity.

The CTV2 was defined as the contrast-enhanced area from pre- and postoperative MRI including a safety margin of 2$3 \mathrm{~cm}$. The margin was further extended to include the surrounding preoperative edema, individually adapted to organs at risk and osseous structures. The PTV2 was generated automatically by adding a $0.5 \mathrm{~cm}$ margin to the CTV 2 and excluding CTV1.
A constant margin of $5 \mathrm{~mm}$ was added circumferentially around the PTV's to account for the penumbra of the radiation beams in 3D-CRT plans

\section{Dose prescription and treatment planning}

For IMRT we used an integrated boost technique. The total dose was 72 Gy, prescribed to the ICRU Reference Point $[23,24]$, resulting in daily fractions of 2.4 Gy for PTV1. A mean dose of $60 \mathrm{~Gy}$ was recommended for PTV2, resulting in daily fractions of $2 \mathrm{~Gy}$.

For 3D-CRT we used a concomitant boost technique. The total dose of $72 \mathrm{~Gy}$ was prescribed to the ICRU Reference Point $[23,24]$. Dose calculations were separated in a dose prescription of 60 Gy for PTV1 and PTV2, and a dose prescription of 12 Gy for PTV1 alone, resulting in equal daily fractions of 2.4 Gy for PTV1 and 2 Gy for PTV2, compared to the integrated boost IMRT technique. Normal tissue dose constraints were 50 Gy (maximum point dose) for chiasm and optic nerves and 50 - 54 Gy for the brainstem.

Table I: Dose constraint values, setted initially for PTV's and OAR's

\begin{tabular}{|c|c|c|c|}
\hline Region of Interest & Type & Target Gy & $\%$ Volume \\
\hline \multirow[t]{3}{*}{ PTVI } & max. dose & 77.04 & - \\
\hline & uniform dose & 72.00 & - \\
\hline & $\min \mathrm{DVH}$ & 68.40 & 95 \\
\hline \multirow[t]{5}{*}{ PTV2 } & uniform dose & 60.00 & - \\
\hline & max. dose & 72.00 & - \\
\hline & $\max . \mathrm{DVH}$ & 67.50 & 5 \\
\hline & $\max . \mathrm{DVH}$ & 64.20 & 15 \\
\hline & $\max . \mathrm{DVH}$ & 63.00 & 25 \\
\hline \multirow[t]{2}{*}{ Brain } & $\max . \mathrm{DVH}$ & 25.00 & 40 \\
\hline & $\max . \mathrm{DVH}$ & 40.00 & 20 \\
\hline \multirow[t]{2}{*}{ Brainstem } & $\max$. Dose & 54.00 & - \\
\hline & $\max . \mathrm{DVH}$ & 50.00 & 30 \\
\hline Chiasma & max. dose & 50.00 & \\
\hline Optic nerves & max. dose & 50.00 & \\
\hline lenses & max. dose & 5.00 & \\
\hline
\end{tabular}


In table 1 the IMRT dose constraint values, setted initially for PTV's and OAR's, are shown.

In all patients the OAR's were outside the PTV's. Despite a hypofractionated setting with single doses in PTV1 by 2.4 Gy the single doses in the OAR's were maximally $2 \mathrm{~Gy}$, corresponding to a conventionally fractionation. So, from a radiobiologically point of view, the established constraints for the OAR's could be taken. The data for normal tissue complication probabilities are those described by Emami [25].

Plans were acceptable for both techniques when the given normal tissue constraints were fulfilled while the mean dose to PTV2 was 60 Gy.

For IMRT we used a step-and-shoot technique and 6-15 $\mathrm{MeV}$ photons for an Elekta Precise $^{\odot}$ linear accelerator (multileaf collimator with leaves projecting to $1 \mathrm{~cm}$ at isocenter). The direct machine parameter optimization (DMPO, Pinnacle $e^{\oplus} 8.0 \mathrm{~m}$ ) algorithm was applied for inverse planning with a $2 \mathrm{~cm}^{2}$ minimum segment area, five minimum segment monitor units and a maximum number of 100 segments. The dose grid size includes the PTV's, organs at risk and scalp and additionally 1-4 cm tissue in all directions. The beam arrangements were determined by the size and location of the tumor and the corresponding PTV's. No restrictions were given for the number of beams or angles or whether noncoplanar beams could be used. For 3D-CRT we used 2-6 beams to cover PTV1 and also PTV2 (table 2).

\section{Plan comparison}

Treatment plan intercomparisons were performed using the following criteria: mean, minimum and maximum doses, Inhomogeneity Index (II), Conformity Index (CI) and Equivalent Uniform Dose (EUD)

Table 2: Summerized plan information

\begin{tabular}{lll}
\hline & \multicolumn{2}{c}{ mean (range) } \\
\hline IMRT & 3D-CRT \\
\hline Monitor Units (MU) & $606(483-845)$ & $482(256-804)$ \\
\hline Beam Number & $7(5-9)$ & $9(4-12)^{*}$ \\
\hline Segments & $91(70-100)$ & - \\
\hline Wedge Number & - & $3(0-5)$ \\
\hline Beam energy (MeV) & $6-15$ & $6-15$ \\
\hline
\end{tabular}

(*summarized beam number for covering PTVI and PTV2)

\section{Inhomogeneity Index (II) and Conformity Index (CI)}

Two indices served to characterize homogeneity and conformity:

$\circ$ Inhomogeneity Index [26] $\mathrm{II}=\left(\mathrm{D}_{\max }-\mathrm{D}_{\min }\right) / \mathrm{D}_{\text {mean }}$

$\mathrm{D}_{\text {max }}$ : maximum PTV dose; $\mathrm{D}_{\min }$ : minimum PTV dose;

$\mathrm{D}_{\text {mean }}$ : mean PTV dose;

○ Conformity Index [27] CI $=\mathrm{PTV}_{\mathrm{PIV}^{2}} / \mathrm{PTV} * \mathrm{PIV}$

PTV $_{\text {PIV }}$ PTV volume covered by $95 \%$ of the prescription dose; PIV: total volume covered by $95 \%$ of the prescription dose.

\section{EUD (Equivalent Uniform Dose)}

The EUD, defined as the biologically equivalent dose that, if given uniformly, will lead to the same effect in the tumor volume or the normal tissues as the actual nonuniform dose distribution, could be, based on Niemierko $[28,29]$, defined as:

$$
\text { EUD }=\left(\frac{1}{N} \sum_{i=1}^{N} d_{i}^{a}\right)^{\frac{1}{a}}
$$

$\mathrm{N}$ : number of voxels in the anatomic structure of interest; $\mathrm{d}_{\mathrm{i}}$ : dose in the i'th voxel; a: tumor or tissue-specific parameter that describes the dose volume effect.

In Pinnacle ${ }^{3}$ IMRT, which is used for EUD-calculation, the equation is slightly modified to allow voxels to be only partially included in a region of interest [30] as:

$$
\text { EUD }=\left(\frac{1}{N} \sum_{i=1}^{N} v_{i} d_{i}^{a}\right)^{\frac{1}{a}}
$$

$\mathrm{v}$ : fraction of the region of interest that is occupied by voxel "i".

In this analysis the tumor or tissue-specific parameter "a", that describes the dose volume effect, was taken, based on Burman, as follows: $\mathrm{a}=-10$ for malignant glioma, $\mathrm{a}=4$ for brain, $\mathrm{a}=6.25$ for brain stem, $\mathrm{a}=4$ for chiasm and optic nerves [31-33].

\section{Statistics}

Statistical analysis was performed using the SPSS 17.0 $\left(\right.$ SPSS $^{\circledast}$, Chicago, Ill) software. The Wilcoxon's matchedpair's test was applied to determine statistical differences between the dose-volume-load calculated with the IB- 
IMRT- versus 3D-CRT-plans and also to determine statistical differences between mean doses and EUD's in the IMRT- and 3D-CRT-plans. Values are expressed as mean \pm standard deviation or as mean value and the range of the values. All p-values reported are two-sided and p $<0.05$ is considered significant.

\section{Results \\ Target subvolume number and shape}

After the described auto-contouring process, based on FET-PET data for PTV1 most patients revealed a complex shape together with multiple subvolumes. Looking on the number of these subvolumes only in 4 patients a sole subvolume was defined, while in 5 and 6 patients 2 and 3 subvolumes appeared, respectively. In one patient a total of 4 subvolumes resulted from the auto-contouring process. In respectively 2, 8 and 6 patients the automatically generated PTV1 had a convex, concave and complex shape (table 3).

Based on the described dose prescription of 72 Gy as point dose to PTV1, patients with a single subvolume $(\mathrm{n}=4)$ had a mean dose of $70.6(69.2-71.5)$ Gy to PTV1. In patients with $3(n=6)$ or $4(n=1)$ subvolumes the mean dose decreased to 67.6 (66.0-68.5) Gy. According to the complexity in the shape of the target volumes an equal decrease of mean dose for PTV1 was observed. For convex shaped PTV1 a mean dose of 71.28 (66.11-73.07) Gy resulted, while in patients with a complex shape the mean dose decreased to 67.70 (59.72-72.99) Gy (table 4, 5).

\section{Inhomogeneity and conformity}

Using the inverse planning technique for IB-IMRT the dose inhomogeneity within PTV1 (HI: 0.17 vs. $0.24, \mathrm{p}=$ 0.02 ) and within PTV2 (HI: 0.34 vs. 0.54, p < 0.01) decreased significantly, compared to 3D-CRT.

The dose conformity for PTV1 (CI: 0.35 vs. 0.14, p < 0.01 )and for PTV2 (CI: 0.64 vs. $0.5, \mathrm{p}<0.01$ ) was significantly improved with IB-IMRT (table 6).

\section{Mean dose, minimum and maximum dose}

The averaged mean dose for PTV2 was slightly, but significantly lower $(60.68 \pm 0.63$ Gy vs. $61.00 \pm 0.78 \mathrm{~Gy}, \mathrm{p}=$ 0.03 ) after inverse treatment planning with IB-IMRT. For PTV1 the mean dose did not differ significantly $(68.76 \pm$ $1.88 \mathrm{~Gy}$ vs. $64.40 \pm 2.79 \mathrm{~Gy}, \mathrm{p}=0.61)$. The minimum dose within PTV1 (61.1 Gy vs. 57.4 Gy, p = 0.02) and within PTV2 (51.4 Gy vs. 40.9 Gy, p < 0.01) increased highly significant after inverse treatment planning. Looking on the dose-volume-load to critical organs only the mean dose to the brain increased significantly (25.6 Gy vs. 22.9 Gy, p < 0.01) (table 7).

\section{EUD}

The averaged mean EUD for PTV2 was significantly improved (59.92 \pm 0.95 Gy vs. $55.3 \pm 4.33$ Gy, p < 0.01) if planned with IB-IMRT vs. 3D-CRT. In addition the EUD for PTV1 was slightly improved $(68.3 \pm 1.93$ Gy vs. $67.3 \pm$ $2.85 \mathrm{~Gy}, \mathrm{p}=0.2$ ) after IB-IMRT. The EUD for the brain was equal with both two planning techniques $(41.7 \pm 3.12 \mathrm{~Gy}$ vs. $41.6 \pm 2.16 \mathrm{~Gy})$ (s. table 8$)$.

Table 3: Target volume characteristics

\begin{tabular}{|c|c|c|}
\hline Target volumes & PTVI (= CTVI *) & $12.1 \pm 18.6 \mathrm{ccm}$ \\
\hline & PTV2 & $175.2 \pm 54.4 \mathrm{ccm}$ \\
\hline \multirow[t]{5}{*}{ PTVI subvolume number } & & patient number \\
\hline & 1 & 4 \\
\hline & 2 & 5 \\
\hline & 3 & 6 \\
\hline & 4 & 1 \\
\hline \multirow{3}{*}{$\begin{array}{l}\text { PTVI geometry } \\
\text { single form and/or subvolume configuration }\end{array}$} & convex & 2 \\
\hline & concave & 8 \\
\hline & complex & 6 \\
\hline \multirow{2}{*}{$\begin{array}{l}\text { Tumor/Brain ratio } \\
\text { (in PTVI) }\end{array}$} & Mean & $2.1(1.7-2.9)$ \\
\hline & $\max$ & $3.3(2.0-4.9)$ \\
\hline
\end{tabular}

(* CTVI is equal to PTVI, s. Target volume definition) 
Table 4: Mean, min. and max. dose (IMRT) in correlation to the subvolume-number in PTVI

\begin{tabular}{|c|c|c|c|c|c|}
\hline & \multicolumn{5}{|c|}{ IMRT } \\
\hline & number of subvolumes & $\mathbf{n}$ & & dose & SD \\
\hline \multirow[t]{12}{*}{ PTV I } & overall & 16 & mean & 68.76 & \pm 1.88 \\
\hline & & & $\min$ & 61.07 & \pm 3.31 \\
\hline & & & $\max$ & 73.14 & \pm 0.98 \\
\hline & I & 4 & mean & 70.60 & \pm 1.01 \\
\hline & & & $\min$ & 63.61 & \pm 3.84 \\
\hline & & & $\max$ & 73.56 & \pm 0.93 \\
\hline & 2 & 5 & mean & 68.50 & \pm 1.91 \\
\hline & & & $\min$ & 60.54 & \pm 3.47 \\
\hline & & & $\max$ & 73.72 & \pm 1.13 \\
\hline & $3 / 4$ & 7 & mean & 67.56 & \pm 0.94 \\
\hline & & & $\min$ & 59.99 & \pm 2.46 \\
\hline & & & $\max$ & 71.94 & \pm 1.32 \\
\hline
\end{tabular}

\section{Discussion}

In malignant gliomas distant tumor spread is rare and more than $80 \%$ of recurrences were found within a rim of $2-3 \mathrm{~cm}$ around the initial tumor site $[34,35]$. Therefore, it seems promising to escalate the radiation dose. In the past, several authors reported improved survival data from non-randomized, retrospective dose escalation trials $[10,36,37]$. These data should be interpreted cautiously because of a potential bias from patient selection [38-40]. In a RTOG multicenter phase-I-trial (RTOG 98-03) dose escalation was conducted using 3D-conformal irradiation [41]. In this trial a four step dose escalation strategy from

Table 5: Mean, min. and max. (IMRT) in correlation to the PTVIconfiguration

\begin{tabular}{|c|c|c|c|c|c|}
\hline & \multicolumn{5}{|c|}{ IMRT } \\
\hline & PTVI-configuration & $\mathbf{n}$ & & dose & (SD) \\
\hline \multirow[t]{12}{*}{ PTV I } & overall & 16 & mean & 68.76 & \pm 1.88 \\
\hline & & & $\min$ & 61.07 & \pm 3.31 \\
\hline & & & $\max$ & 73.14 & \pm 0.98 \\
\hline & Convex & 2 & mean & 71.28 & \pm 0.35 \\
\hline & & & $\min$ & 66.11 & \pm 3.56 \\
\hline & & & $\max$ & 73.05 & \pm 0.72 \\
\hline & Concave & 8 & mean & 68.66 & \pm 1.79 \\
\hline & & & $\min$ & 60.82 & \pm 3.31 \\
\hline & & & $\max$ & 72.81 & \pm 1.85 \\
\hline & complex & 6 & mean & 67.70 & \pm 0.98 \\
\hline & & & $\min$ & 59.72 & \pm 1.58 \\
\hline & & & $\max$ & 72.99 & \pm 1.03 \\
\hline
\end{tabular}

66 to 84 Gy was applied and median survival increased from 11.6 to 19.3 months in patients with a boost target volume smaller than $75 \mathrm{ccm}$. However, with boost target volumes $\geq 75 \mathrm{ccm}$ the improvement was markedly smaller (8.2 vs. 13.9 months). No benefit was seen in progression free survival.

None of the randomized trials could demonstrate an improvement in median survival after locally restricted dose escalation. Souhami used a stereotactic boost technique [11], Laperriere [42] and Selker [43] used brachytherapy in addition to external beam radiotherapy with 60 Gy. Souhami addressed, that the results from the RTOG 93-05 trial were not completely surprising, because glioblastomas are inherently infiltrating neoplasms. Considering that delineation of tumor volumes in treatment planning was based on morphological imaging, they discussed, that "biopsy and magnetic resonance spectroscopy analyses have demonstrated significant microscopic tumor extension beyond the contrast-enhancing lesion, thereby limiting the effectiveness of focal radiotherapy".

MRI is highly sensitive in detecting brain tissue abnormalities. In a biopsy-controlled trial Pauleit obtained a 96\% sensitivity to detect glioma tissue [15]. But the specificity was only $53 \%$. Better tumor brain delineation became possible with the use of PET (Positron-Emission-Tomography) imaging with radio-labeled amino acids, like O-(2F-18-Fluorethyl)-L-Tyrosin (FET) [18]. The use of FET-PET in addition to MRI yields a sensitivity of $93 \%$, similar to MRI alone, but a markedly improved specificity of $94 \%$ [15].

Therefore, it is straightforward to integrate FET-PET imaging in dose escalation irradiation strategies. Several authors could already demonstrate the feasibility of this approach and mentioned the estimated positive impact [44-46]. The process of automatic delineation of the PETpositive area as biological target volume, done in our planning study, prevents the known problem of interobserver variations [47] but leads to a pronounced irregularity in target volume shape. After auto-contouring the PETpositive areas in our study with a cut-off value of 1.6, $37.5 \%$ of the patients revealed a very complex target shape, comprising multiple separate sub volumes, half of them cuttlefish-like shaped and mostly arranged around the surgical cave.

Irradiation with intensity-modulated dose application led to an improvement in target coverage compared with 3DCRT in different tumor entities, i.e. head and neck [48], lung [49], breast [50], prostate [32,51,52] or other [53]. For radiotherapy of glioblastomas the feasibility and efficacy of IMRT planning with a simultaneous boost could be shown by Chan et al. [54]. Narayana et al. found no 
Table 6: Inhomogeneity Index and Conformity Index for PTVI and 2 in IMRT versus 3D-CRT

\begin{tabular}{llcccccccccc}
\hline & \multicolumn{3}{c}{ Inhomogeneity Index } & & & \multicolumn{3}{c}{ Conformity Index } \\
\hline & \multicolumn{3}{c}{ IMRT } & \multicolumn{2}{c}{ 3D-CRT } & P & & IMRT & & 3D-CRT & P \\
\hline & Mean & SD & mean & SD & & mean & SD & mean & SD & & \\
\hline PTVI & 0.17 & \pm 0.05 & 0.24 & \pm 0.12 & $\mathbf{0 . 0 2}$ & 0.35 & \pm 0.12 & 0.14 & \pm 0.1 & $<0.01$ \\
\hline PTV2 & 0.34 & \pm 0.54 & 0.54 & \pm 0.13 & $<0.01$ & 0.64 & \pm 0.07 & 0.50 & \pm 0.13 & $<0.01$ \\
\hline
\end{tabular}

improvement in target coverage using IMRT in high-grade gliomas in comparison with 3D-CRT. Nevertheless, the normal brain, which received a dose of $\geq 18$ and $\geq 24$ Gy as well as the mean dose to the brainstem could be reduced with IMRT [55]. In contrast, MacDonald demonstrated in a similar analysis an improved target coverage and also confirmed reduced radiation dose to the brain, brainstem and optic chiasm [56]. Also Hermanto showed an improved target conformity using an IMRT vs. 3D-CRT planning for high-grade gliomas [57]. A locally restricted integrated dose escalation was not considered in these analyses.

In our setting, using an integrated complex boost volume a significantly better conformity could be achieved with

Table 7: Mean, min., and max. doses for PTV's and OAR's in IMRT versus 3D-CRT

\begin{tabular}{|c|c|c|c|c|c|c|}
\hline & & \multicolumn{2}{|c|}{ IMRT } & \multicolumn{2}{|c|}{ 3D-CRT } & \multirow[t]{2}{*}{$\mathbf{p}$} \\
\hline & & Dose & SD & dose & SD & \\
\hline \multirow[t]{3}{*}{ PTV I } & mean & 68.76 & \pm 1.88 & 64.40 & \pm 2.79 & 0.61 \\
\hline & $\min$ & 61.07 & \pm 3.31 & 57.39 & \pm 6.79 & 0.02 \\
\hline & $\max$ & 73.14 & \pm 0.98 & 73.94 & \pm 1.88 & 0.1 \\
\hline \multirow[t]{3}{*}{ PTV 2} & mean & 60.68 & \pm 0.63 & 61.00 & \pm 0.78 & 0.03 \\
\hline & $\min$ & 51.40 & \pm 3.44 & 40.89 & \pm 7.03 & $<0.01$ \\
\hline & $\max$ & 71.90 & $\pm|.5|$ & 73.68 & \pm 2.64 & 0.01 \\
\hline Brain & Mean & 25.57 & \pm 3.24 & 22.90 & \pm 4.31 & $<0.01$ \\
\hline \multirow[t]{2}{*}{ Brainstem } & mean & 13.76 & \pm 8.74 & 13.37 & \pm 9.25 & 0.79 \\
\hline & $\max$ & 37.04 & \pm 20.2 & 36.56 & \pm 20.15 & 0.77 \\
\hline \multirow[t]{2}{*}{ Chiasm } & mean & $|8.5|$ & \pm 12.56 & 15.83 & \pm 13.33 & 0.14 \\
\hline & $\max$ & 28.16 & \pm 17.9 & 23.56 & \pm 16.84 & 0.07 \\
\hline \multirow[t]{2}{*}{ Optic nerve rt. } & mean & 8.48 & \pm 6.49 & 7.57 & \pm 8.66 & 0.64 \\
\hline & $\max$ & 15.2 & \pm 12.04 & 12.19 & \pm 12.43 & 0.14 \\
\hline \multirow{2}{*}{ Optic nerve It. } & mean & 13.02 & \pm 11.94 & 13.50 & \pm 14.33 & 0.79 \\
\hline & $\max$ & 18.99 & \pm 16.39 & 18.20 & \pm 17.69 & 0.69 \\
\hline
\end{tabular}

IMRT for both planning target volumes, PTV1 (0.35 vs. $0.14 ; \mathrm{p}<0.01)$ and PTV2 (0.64 vs. $0.5, \mathrm{p}<0.01)$. The dose inhomogeneities for PTV1 and PTV2 decreased significantly with IMRT (table 6, figure $1 \mathrm{a}, \mathrm{b}$ ).

In addition to the prescribed dose within PTV1 a dose of 60 Gy as mean dose for PTV2 was required as equally rated first level priority. In contrast to the ICRU 50/62 reports $[23,24]$, which limits the recommended dose range between 95 and 107\% of the prescribed dose to PTV2, a dose of $120 \%$ was accepted as essential default value to achieve a point dose prescription of 72 Gy within PTV1. To limit at least the integral dose to PTV2 a mean dose of 60 Gy with a minimum dose of $95 \%$ - as second level priority - was required for plan acceptance. The resulting mean doses for PTV2 were acceptable for IBIMRT planning (60.68 Gy \pm 0.63$)$ and 3D-CRT planning (61.00 Gy \pm 0.78$)$. Using a prescription dose of $72 \mathrm{~Gy}$ as point dose to PTV1, the mean dose to PTV1 was less than the prescribed dose in all patients. After 3D-CRT planning a mean dose of $64.4 \pm 2.79$ Gy could be obtained and after IMRT planning the mean dose averaged over all patients was $68.76 \pm 1.88$ Gy to PTV1 (table 7 ).

Using an integrated boost technique for patients with high-grade gliomas, Thilmann could deliver an escalated mean dose of $75 \mathrm{~Gy}$ to the enhancing lesion in MRI (PTV1) and a mean dose of 60 Gy to the surrounding clinical risk area (PTV2) [58]. The authors allowed a dose delivery of more than $107 \%$ of the prescribed dose to $13.9 \%$ of the PTV2 volume. The maximum dose constraints for chiasm and optic nerves (52 Gy) were slightly increased compared to our study. A marked difference to our study was the MRI based delineation of PTV1, resulting in convex shaped singular target volumes. The shape and number of subvolumes of auto-contoured target volumes in our study was markedly more complex and had an impact on the mean dose value for PTV1 (tables 4 and 5 , figures $2 \mathrm{a}, \mathrm{b}, 3 \mathrm{a}, \mathrm{b}, 4 \mathrm{a}, \mathrm{b})$. In patients with a singular PTV1 $(\mathrm{n}=4)$ a mean dose of 70.6 Gy was achievable. But in patients with 3 and 4 subvolumes of PTV1 the mean dose decreased to 67.6 Gy. 
Table 8: Mean dose and EUD for PTV's and OAR's in IMRT versus 3D-CRT

\begin{tabular}{|c|c|c|c|c|c|c|c|c|c|c|}
\hline & \multicolumn{5}{|c|}{ Mean Dose } & \multicolumn{5}{|c|}{ EUD } \\
\hline & \multicolumn{2}{|c|}{ IMRT } & \multicolumn{2}{|c|}{ 3D-CRT } & \multirow[t]{2}{*}{$\mathbf{p}$} & \multicolumn{2}{|c|}{ IMRT } & \multicolumn{2}{|c|}{ 3D-CRT } & \multirow[t]{2}{*}{$\mathbf{p}$} \\
\hline & mean & SD & mean & SD & & mean & SD & mean & SD & \\
\hline PTV I & 68.76 & \pm 1.88 & 64.40 & \pm 2.79 & 0.61 & 68.34 & \pm 1.93 & 67.29 & \pm 2.85 & 0.2 \\
\hline PTV 2 & 60.68 & \pm 0.63 & 61.00 & \pm 0.78 & 0.03 & 59.92 & \pm 0.95 & 55.30 & \pm 4.33 & $<0.01$ \\
\hline Brain & 25.57 & \pm 3.24 & 22.90 & \pm 4.31 & $<0.01$ & 41.57 & \pm 2.16 & 41.73 & \pm 3.12 & 0.69 \\
\hline Brainstem & 13.76 & \pm 8.74 & 13.37 & \pm 9.25 & 0.79 & 21.83 & \pm 11.91 & 22.49 & \pm 12.94 & 0.48 \\
\hline Chiasm & $|8.5|$ & \pm 12.56 & 15.83 & \pm 13.33 & 0.14 & 19.64 & \pm 13.2 & 16.95 & $\pm|3.7|$ & 0.12 \\
\hline Optic nerve rt. & 8.48 & \pm 6.49 & 7.57 & \pm 8.66 & 0.64 & 10.2 & \pm 7.76 & 8.81 & \pm 9.49 & 0.41 \\
\hline Optic nerve It. & 13.02 & \pm 16.94 & 13.50 & \pm 14.33 & 0.79 & 14.07 & \pm 12.74 & 14.57 & \pm 14.65 & 0.78 \\
\hline
\end{tabular}
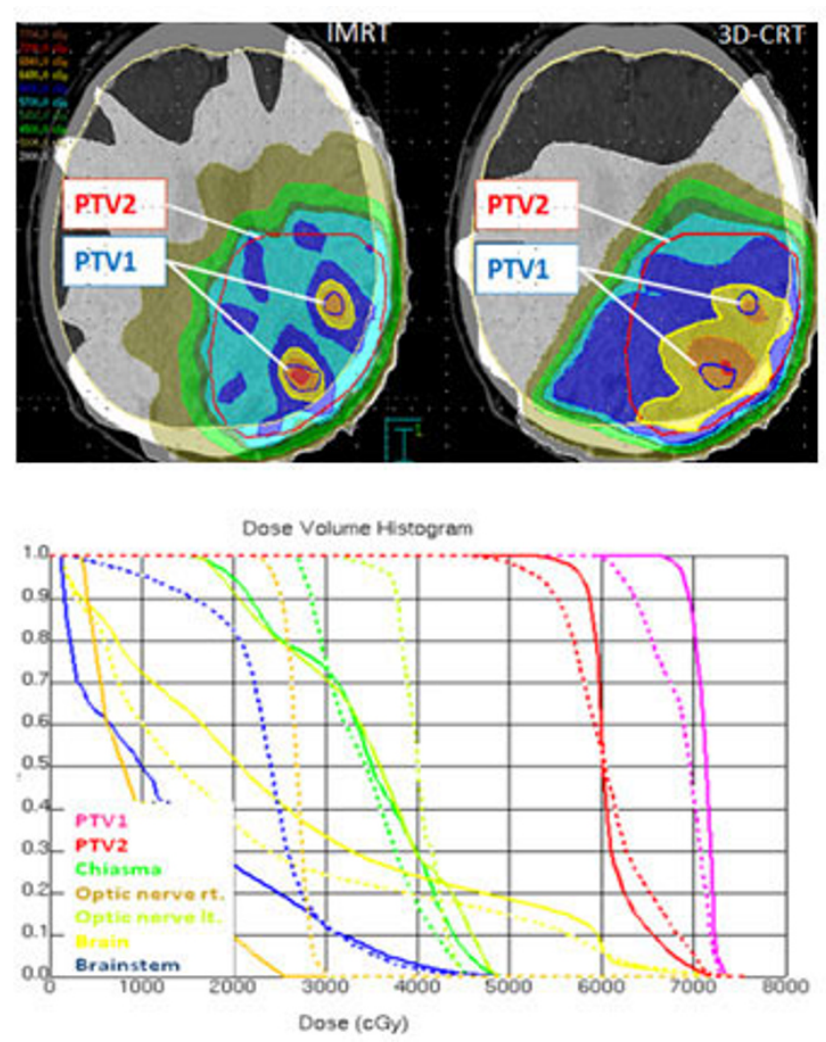

Figure I

a) Isodose distribution (dose wash) for IMRT and 3DCRT-planning. b) Dose-volume-histograms for IMRT and 3D-CRT in comparison (IMRT: aligned, 3D- CRT: dashed).

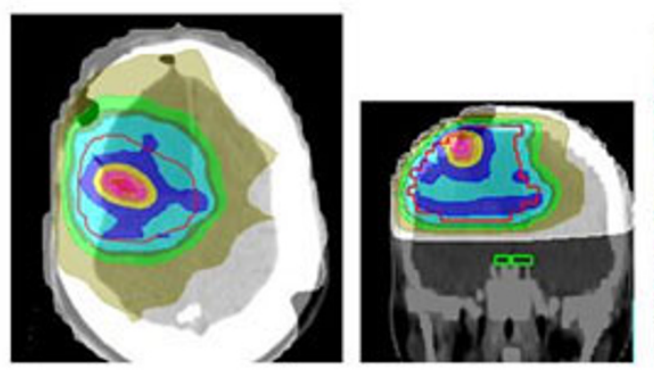

Absolute dose

7704.0 cGy

$7200.0 \mathrm{cGY}$

$6840.0 \mathrm{CGY}$

$6000.0 \mathrm{cGy}$

5700.0 cGY

$5400.0 \mathrm{cGy}$

4500.0 cGY

3000.0 cGy

2000.0 cGY

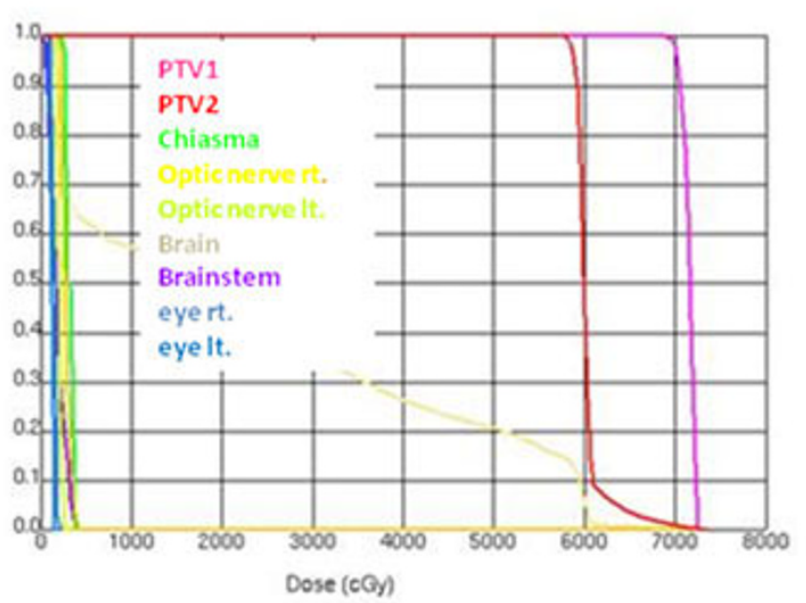

Figure 2

a) Dose wash for IMRT. Explanation of a convex configuration of PTVI (one FET- subvolume) with a mean dose of 70.5 Gy. b) Dose-volume-histogram (IMRT) for a convex configuration of PTVI (one FET-subvolume) with a mean dose of $70.5 \mathrm{~Gy}$. 
The averaged minimum dose to PTV2 after 3D-CRT planning was $40.9 \mathrm{~Gy}$ (68\% of the required dose) and thus not acceptable for most of the patients. After IB-IMRT planning the mean minimum dose to PTV2 increased to 51.4 Gy ( $86 \%$ of the required dose). The minimum dose was located $1.5-2 \mathrm{~cm}$ distant to the contrast-enhanced area in MRI and thus acceptable for treatment $[41,59]$. According to Tome and Fowler a minimum dose or "cold dose" lower than the prescribed dose by substantially more than $10 \%$ can be detrimental in tumor control [60]. In addition, Niemierko emphasized, that a "cold spot" cannot be compensated by any dose delivered to the rest of the target volume [28]. Unlike the mean dose, the equivalent uniform dose (EUD) concept includes the impact of dose inhomogeneities and volumetric effect [61]. The EUD is the homogeneous dose inside an organ that has the same clinical effect as a given, arbitrary dose distribution [62]. The EUD concept allows reducing a complex three-dimensional dose distribution into a single metric value $[33,62]$. Niemierko pointed out, that for relatively small dose
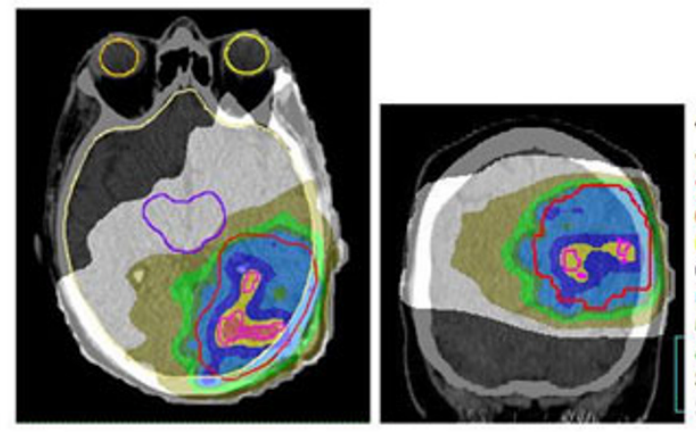

Absolute dose $7704.0 \mathrm{cGy}$ $7200.0 \mathrm{cGY}$ $6840.0 \mathrm{cGY}$ $6000.0 \mathrm{cGy}$ $5700.0 \mathrm{cGY}$ $5400.0 \mathrm{cGY}$ $4500.0 \mathrm{cGY}$ $3000.0 \mathrm{cGY}$

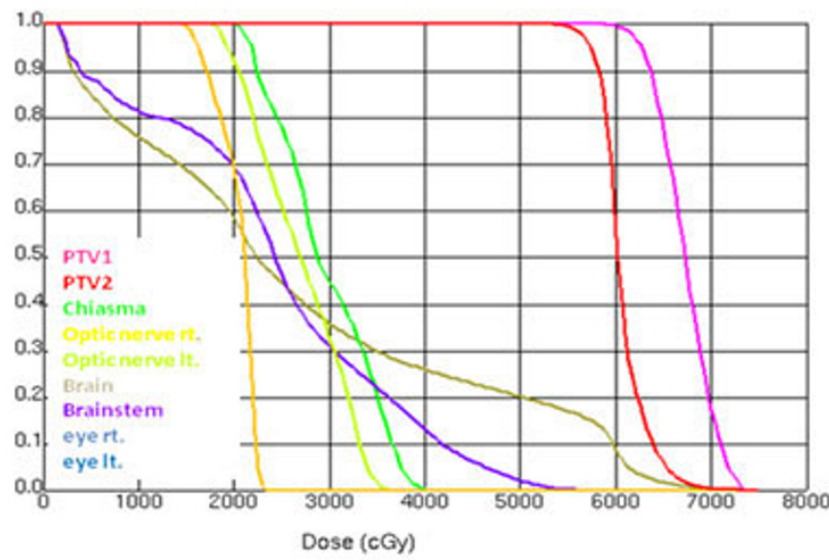

Figure 3

a) Isodose distribution (dose wash) for IMRT. Explanation of a concave configurationof PTVI (3 FET-subvolumes) with a mean dose of $68.0 \mathrm{~Gy}$. b)Dose-volume-histogram (IMRT) for a concave configuration of PTVI (3 FETsubvolumes) with a mean dose of $68.0 \mathrm{~Gy}$. inhomogeneities the mean dose might be a good approximation to EUD [28]. Furthermore, the authors explained that the minimum target dose can significantly underestimate the dose actually delivered, if the cold spot is very small. Considering the EUD concept, marked differences were evident in our study for PTV2. The EUD for PTV2 after 3D-CRT was significantly lower (55.3 Gy and 59.92 Gy, $\mathrm{p}<0.01$ ), while for PTV1 no significant difference was obtained.

For the OAR's only the EUD values for the brainstem differed significantly (25.6 Gy IB-IMRT; 22.9 Gy 3D-CRT, p $<0.01$ ) (table 8 ). In contrast to the increase in mean dose, the EUD values for the brain were not significantly different (41.6 Gy for IB-IMRT and 41.7 Gy for 3D-CRT ( $\mathrm{p}=$ $0.7))$.
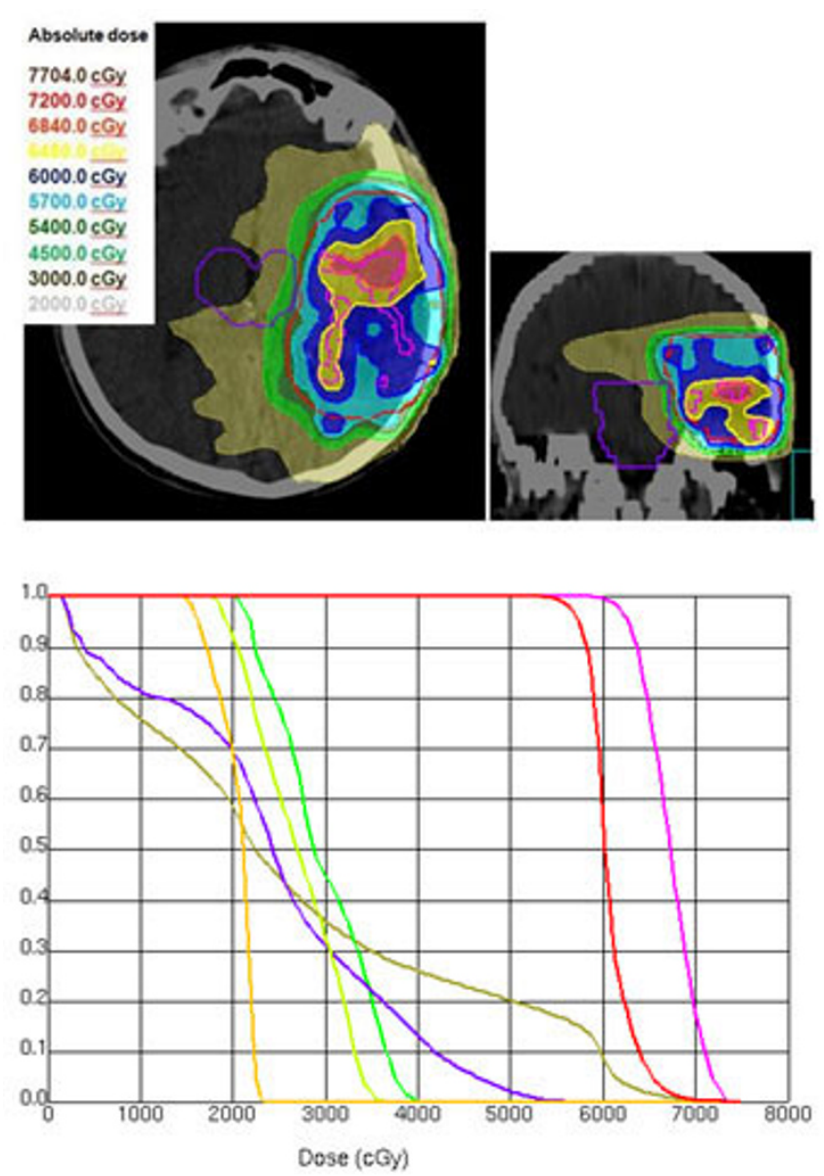

Figure 4

a) Isodose distribution (dose wash) for IMRT. Explanation of a complex. configuration of PTVI (with 2 FET-subvolumes) with a mean dose of 67.2 Gy. b) Dose-volumehistogram (IMRT) for a complex configuration of PTVI (with 2 FET-subvolumes) with a mean dose of 67.2 Gy. 


\section{Conclusion}

Auto-contouring of the integrated boost volume resulted in complex target volume shapes. With the given constraints, the dose prescription to PTV1 (72 Gy), combined with a limited mean dose to PTV2 (60 Gy) could be achieved. Nevertheless a mean dose of 72 Gy to PTV1 could not be realized, neither with 3D-CRT nor with the IB-IMRT. Comparing both techniques, IB-IMRT provided several improvements. IB-IMRT led to a significantly better homogeneity and conformity, compared to 3D-CRT. The mean dose and the EUD values for PTV2 were inacceptable low with 3D-CRT, which would decrease tumor control. The EUD concept seems to be very useful for inverse planning, because the complex dose distribution can be reduced to one single parameter and also volume parameters and biological effects are taken into account. Further plan comparisons are simplified. The prognostic impact of this technique based on MR- and FET-PET imaging for patients with glioblastomas will be evaluated in an ongoing prospective phase-II trial in our clinic.

\section{Competing interests}

The authors declare that they have no competing interests.

\section{Authors' contributions}

MDP has made substantial contributions to the conception, acquisition of data, analysis and interpretation of data and drafted the manuscript. MP has been involved in acquisition of data and revised the manuscript. RH has been involved in acquisition of data and revised the manuscript. GS has been involved in acquisition of data and revised the manuscript. $\mathrm{CD}$ has been involved in acquisition of data and revised the manuscript. CA has been involved in acquisition of data and revised the manuscript. HJK has been involved in acquisition of data and revised the manuscript. KJL has made substantial contributions to the conception, acquisition of data, analysis and interpretation of data and revised the manuscript. MJE has made substantial contributions to the conception, acquisition of data, analysis and interpretation of data and revised the manuscript.

\section{Acknowledgements}

We would like to thank the staff who took care of our patients' needs, and who were involved in gathering, documenting, verifying, forwarding and processing the clinical data.

\section{References}

I. Stupp R, Mason WP, Bent MJ van den, Weller M, Fisher B, Taphoorn MJ, et al:: Radiotherapy plus concomitant and adjuvant temozolomide for glioblastoma. N EnglJ Med 2005, 352(I 0):987-996.

2. Stupp R, Hegi ME, Mason WP, Bent MJ van den, Taphoorn MJ, Janzer $\mathrm{RC}$, et al.: Effects of radiotherapy with concomitant and adjuvant temozolomide versus radiotherapy alone on survival in glioblastoma in a randomised phase III study: 5-year analysis of the EORTC-NCIC trial. Lancet Oncol 2009, 10(5):459-466.
3. Bleehen NM, Stenning SP: A Medical Research Council trial of two radiotherapy doses in the treatment of grades 3 and 4 astrocytoma. The Medical Research Council Brain Tumour Working Party. Br J Cancer 1991, 64(4):769-774.

4. Walker MD, Strike TA, Sheline GE: An analysis of dose-effect relationship in the radiotherapy of malignant gliomas. Int $J$ Radiat Oncol Biol Phys 1979, 5(10): 1725-1731.

5. Taghian A, Ramsay J, Allalunis-Turner J, Budach W, Gioioso D, Pardo $\mathrm{F}$, et al.: Intrinsic radiation sensitivity may not be the major determinant of the poor clinical outcome of glioblastoma multiforme. Int J Radiat Oncol Biol Phys 1993, 25(2):243-249.

6. Taghian A, DuBois W, Budach W, Baumann M, Freeman J, Suit H: In vivo radiation sensitivity of glioblastoma multiforme. Int J Radiat Oncol Biol Phys 1995, 32(I):99-104.

7. Taghian $A$, Suit $H$, Baumann $M$ : In vitro and in vivo radiation sensitivity of glioblastoma multiforme: correction. Int J Radiat Oncol Biol Phys 1996, 35(5): I I 24- I I 25.

8. Taghian A: In vitro and in vivo radiation sensitivity of glioblastoma multiforme: correction. Int J Radiat Oncol Biol Phys 1998, 42(2):464.

9. Loeffler JS, Alexander E III, Shea WM, Wen PY, Fine HA, Kooy HM, et al: Radiosurgery as part of the initial management of patients with malignant gliomas. J Clin Oncol 1992, I0(9): 1379-1385.

10. Sarkaria JN, Mehta MP, Loeffler JS, Buatti JM, Chappell RJ, Levin AB, et al:: Radiosurgery in the initial management of malignant gliomas: survival comparison with the RTOG recursive partitioning analysis. Radiation Therapy Oncology Group. Int J Radiat Oncol Biol Phys 1995, 32(4):93I-94I.

II. Souhami L, Seiferheld W, Brachman D, Podgorsak EB, Werner-Wasik $M$, Lustig R, et al.: Randomized comparison of stereotactic radiosurgery followed by conventional radiotherapy with carmustine to conventional radiotherapy with carmustine for patients with glioblastoma multiforme: report of Radiation Therapy Oncology Group 93-05 protocol. Int J Radiat Oncol Biol Phys 2004, 60(3):853-860.

12. Grosu AL, Weber WA, Franz M, Stark S, Piert M, Thamm R, et al.: Reirradiation of recurrent high-grade gliomas using amino acid PET (SPECT)/CT/MRI image fusion to determine gross tumor volume for stereotactic fractionated radiotherapy. Int J Radiat Oncol Biol Phys 2005, 63(2):5 I I-5I9.

13. Lee IH, Piert M, Gomez-Hassan D, Junck L, Rogers L, Hayman J, et al: Association of I I C-methionine PET uptake with site of failure after concurrent temozolomide and radiation for primary glioblastoma multiforme. Int J Radiat Oncol Biol Phys 2009, 73(2):479-485.

14. Ogawa T, Shishido F, Kanno I, Inugami A, Fujita H, Murakami M, et al:: Cerebral glioma: evaluation with methionine PET. Radiology 1993, 186(I):45-53.

15. Pauleit D, Floeth F, Hamacher K, Riemenschneider MJ, Reifenberger G, Muller HW, et al:: $\mathbf{O}-(2-[18 F]$ fluoroethyl)-L-tyrosine PET combined with MRI improves the diagnostic assessment of cerebral gliomas. Brain 2005, I 28(Pt 3):678-687.

16. Rachinger W, Goetz C, Popperl G, Gildehaus FJ, Kreth FW, Holtmannspotter $M$, et al.: Positron emission tomography with $\mathbf{O}$ (2-[I8F]fluoroethyl)-I-tyrosine versus magnetic resonance imaging in the diagnosis of recurrent gliomas. Neurosurgery 2005, 57(3):505-5II.

17. Fuss M, Salter BJ, Rassiah P, Cheek D, Cavanaugh SX, Herman TS: Repositioning accuracy of a commercially available doublevacuum whole body immobilization system for stereotactic body radiation therapy. Technol Cancer Res Treat 2004, 3(I):59-67.

18. Langen KJ, Hamacher K, Weckesser M, Floeth F, Stoffels G, Bauer D, et al.: O-(2-[18F]fluoroethyl)-L-tyrosine: uptake mechanisms and clinical applications. Nucl Med Biol 2006, 33(3):287-294.

19. Roesch P, Netsch T, McNutt T, Shoenbill J, Roost P: Syntegra Automated image registration algorithms. Philips White Paper 2003.

20. Weckesser M, Griessmeier M, Schmidt D, Sonnenberg F, Ziemons K, Kemna $L$, et al.: lodine- $\mathbf{2 3}$ alpha-methyl tyrosine single-photon emission tomography of cerebral gliomas: standardised evaluation of tumour uptake and extent. Eur J Nucl Med 1998, 25(2): 150-156. 
21. Popperl G, Gotz C, Rachinger W, Gildehaus FJ, Tonn JC, Tatsch K: Value of O-(2-[18F]fluoroethyl)- L-tyrosine PET for the diagnosis of recurrent glioma. Eur J Nucl Med Mol Imaging 2004 3 I (I I): I 464-I 470.

22. Weckesser M, Langen $\mathrm{KJ}$, Rickert $\mathrm{CH}$, Kloska S, Straeter $\mathrm{R}$, Hamacher K, et al:: O-(2-[ [ 8F]fluorethyl)-L-tyrosine PET in the clinical evaluation of primary brain tumours. Eur J Nucl Med Mol Imaging 2005, 32(4):422-429.

23. Prescribing, Recording and Reporting Photon Beam Therapy. Journal of the International Commission on Radiation Units and Measurements 1993. Report 50

24. Prescribing, Recording and Reporting Photon Beam Therapy (Supplement to ICRU $\mathbf{5 0}$ Report). Journal of the International Commission on Radiation Units and Measurements 1999. Report 62

25. Emami B, Lyman J, Brown A, Coia L, Goitein M, Munzenrider JE, et al: Tolerance of normal tissue to therapeutic irradiation. Int J Radiat Oncol Biol Phys 199I, 21(1):109-122.

26. Tome WA, Meeks SL, Buatti JM, Bova FJ, Friedman WA, Li Z: A highprecision system for conformal intracranial radiotherapy. Int J Radiat Oncol Biol Phys 2000, 47(4): I | 37-I I 43.

27. Paddick I: A simple scoring ratio to index the conformity of radiosurgical treatment plans. Technical note. J Neurosurg 2000, 3:219-222.

28. Niemierko A: Reporting and analyzing dose distributions: a concept of equivalent uniform dose. Med Phys 1997, 24(I): $103-110$.

29. Niemierko A: Radiobiological models of tissue response to radiation in treatment planning systems. Tumori 1998, 84(2): | $40-143$.

30. RaySearch Laboratories $A B$ SS: Biological optimization using the equivalent uniform dose (EUD) in Pinnacle ${ }^{3}$. RaySearch White Paper 2003. WP-EUD rev.I, 0310

31. Burman C, Kutcher GJ, Emami B, Goitein M: Fitting of normal tissue tolerance data to an analytic function. Int $\int$ Radiat Oncol Biol Phys 1991, 21(1): 123-135

32. Luxton G, Keall PJ, King CR: A new formula for normal tissue complication probability (NTCP) as a function of equivalent uniform dose (EUD). Phys Med Biol 2008, 53(I):23-36.

33. Semenenko VA, Reitz B, Day E, Qi XS, Miften M, Li XA: Evaluation of a commercial biologically based IMRT treatment planning system. Med Phys 2008, 35(I 2):585 I-5860.

34. Hochberg FH, Pruitt A: Assumptions in the radiotherapy of glioblastoma. Neurology 1980, 30(9):907-91I.

35. Jansen EP, Dewit LG, van HM, Bartelink $H$ : Target volumes in radiotherapy for high-grade malignant glioma of the brain. Radiother Oncol 2000, 56(2): $15 \mid-156$.

36. Shrieve DC, Alexander E III, Black PM, Wen PY, Fine HA, Kooy HM, et al: Treatment of patients with primary glioblastoma multiforme with standard postoperative radiotherapy and radiosurgical boost: prognostic factors and long-term outcome. J Neurosurg 1999, 90(I):72-77.

37. Tanaka M, Ino Y, Nakagawa K, Tago M, Todo T: High-dose conformal radiotherapy for supratentorial malignant glioma: a historical comparison. Lancet Oncol 2005, 6(I 2):953-960.

38. Irish WD, Macdonald DR, Cairncross JG: Measuring bias in uncontrolled brain tumor trials--to randomize or not to randomize? Can J Neurol Sci 1997, 24(4):307-3I2.

39. Litofsky NS, Bauer AM, Kasper RS, Sullivan CM, Dabbous OH: Image-guided resection of high-grade glioma: patient selection factors and outcome. Neurosurg Focus 2006, 20(4):EI6.

40. Winger MJ, Macdonald DR, Schold SC Jr, Cairncross JG: Selection bias in clinical trials of anaplastic glioma. Ann Neurol 1989 26(4):53I-534.

4I. Tsien C, Moughan J, Michalski JM, Gilbert MR, Purdy J, Simpson J, et al.: Phase I three-dimensional conformal radiation dose escalation study in newly diagnosed glioblastoma: Radiation Therapy Oncology Group Trial 98-03. Int J Radiat Oncol Biol Phys 2009, 73(3):699-708.

42. Laperriere NJ, Leung PM, McKenzie S, Milosevic M, Wong S, Glen J, et al.: Randomized study of brachytherapy in the initial management of patients with malignant astrocytoma. Int J Radiat Oncol Biol Phys 1998, 4I(5): 1005-10II.

43. Selker RG, Shapiro WR, Burger P, Blackwood MS, Arena VC, Gilder JC, et al.: The Brain Tumor Cooperative Group NIH Trial 87-
0I: a randomized comparison of surgery, external radiotherapy, and carmustine versus surgery, interstitial radiotherapy boost, external radiation therapy, and carmustine. Neurosurgery 2002, 5 I (2):343-355.

44. Rickhey M, Koelb O, Eilles C, Bogner L: A biologically adapted dose-escalation approach, demonstrated for I8F-FET-PET in brain tumors. Strahlenther Onkol 2008, 184(10):536-542.

45. Vees H, Senthamizhchelvan S, Miralbell R, Weber DC, Ratib O, Zaidi $\mathrm{H}$ : Assessment of various strategies for I8F-FET PET-guided delineation of target volumes in high-grade glioma patients. Eur J Nucl Med Mol Imaging 2009, 36(2): $182-193$

46. Weber DC, Zilli T, Buchegger F, Casanova N, Haller G, Rouzaud M, et al:: [(18)F]Fluoroethyltyrosine- positron emission tomography-guided radiotherapy for high-grade glioma. Radiat Oncol 2008, 3:44.

47. Weltens C, Menten J, Feron M, Bellon E, Demaerel P, Maes F, et al:: Interobserver variations in gross tumor volume delineation of brain tumors on computed tomography and impact of magnetic resonance imaging. Radiother Oncol 200I, 60(I):49-59.

48. Cozzi L, Fogliata A, Bolsi A, Nicolini G, Bernier J: Three-dimensional conformal vs. intensity-modulated radiotherapy in head-and-neck cancer patients: comparative analysis of dosimetric and technical parameters. Int J Radiat Oncol Biol Phys 2004, 58(2):617-624

49. Grills IS, Yan D, Martinez AA, Vicini FA, Wong JW, Kestin LL: Potential for reduced toxicity and dose escalation in the treatment of inoperable non-small-cell lung cancer: a comparison of intensity-modulated radiation therapy (IMRT), 3D conformal radiation, and elective nodal irradiation. Int J Radiat Oncol Biol Phys 2003, 57(3):875-890.

50. Selvaraj RN, Beriwal S, Pourarian RJ, Lalonde RJ, Chen A, Mehta K, et al.: Clinical implementation of tangential field intensity modulated radiation therapy (IMRT) using sliding window technique and dosimetric comparison with 3D conformal therapy (3DCRT) in breast cancer. Med Dosim 2007, 32(4):299-304.

5I. Pinkawa M, Siluschek J, Gagel B, Piroth MD, Demirel C, Asadpour B et al.: Postoperative radiotherapy for prostate cancer: evaluation of target motion and treatment techniques (intensitymodulated versus conformal radiotherapy). Strahlenther Onkol 2007, I83(I):23-29.

52. Pinkawa M, Attieh C, Piroth MD, Holy R, Nussen S, Klotz J, et al: Dose-escalation using intensity-modulated radiotherapy for prostate cancer--evaluation of the dose distribution with and without 18F-choline PET-CT detected simultaneous integrated boost. Radiother Oncol 2009, 93(2):213-219.

53. Pirzkall A, Carol M, Lohr F, Hoss A, Wannenmacher M, Debus J: Comparison of intensity-modulated radiotherapy with conventional conformal radiotherapy for complex-shaped tumors. Int J Radiat Oncol Biol Phys 2000, 48(5): I 37 I- 1380.

54. Chan MF, Schupak K, Burman C, Chui CS, Ling CC: Comparison of intensity-modulated radiotherapy with three-dimensional conformal radiation therapy planning for glioblastoma multiforme. Med Dosim 2003, 28(4):26I-265.

55. Narayana A, Yamada J, Berry S, Shah P, Hunt M, Gutin PH, et al: Intensity-modulated radiotherapy in high-grade gliomas: clinical and dosimetric results. Int J Radiat Oncol Biol Phys 2006, 64(3):892-897.

56. MacDonald SM, Ahmad S, Kachris S, Vogds BJ, DeRouen M, Gittleman $A E$, et al.: Intensity modulated radiation therapy versus threedimensional conformal radiation therapy for the treatment of high grade glioma: a dosimetric comparison. J Appl Clin Med Phys 2007, 8(2):47-60.

57. Hermanto U, Frija EK, Lii MJ, Chang EL, Mahajan A, Woo SY: Intensity-modulated radiotherapy (IMRT) and conventional three-dimensional conformal radiotherapy for high-grade gliomas: does IMRT increase the integral dose to normal brain? Int J Radiat Oncol Biol Phys 2007, 67(4): I I35-I I 44.

58. Thilmann C, Zabel A, Grosser KH, Hoess A, Wannenmacher M, Debus J: Intensity-modulated radiotherapy with an integrated boost to the macroscopic tumor volume in the treatment of high-grade gliomas. Int J Cancer 200I, 96(6):34I-349.

59. Chang EL, Akyurek S, Avalos T, Rebueno N, Spicer C, Garcia J, et al.: Evaluation of peritumoral edema in the delineation of radio- 
therapy clinical target volumes for glioblastoma. Int J Radiat Oncol Biol Phys 2007, 68(I): 144-150.

60. Tome WA, Fowler JF: On cold spots in tumor subvolumes. Med Phys 2002, 29(7): 1590-I598.

61. Qi XS, Schultz CJ, Li XA: An estimation of radiobiologic parameters from clinical outcomes for radiation treatment planning of brain tumor. Int J Radiat Oncol Biol Phys 2006, 64(5): $1570-1580$.

62. Thieke C, Bortfeld T, Niemierko A, Nill S: From physical dose constraints to equivalent uniform dose constraints in inverse radiotherapy planning. Med Phys 2003, 30(9):2332-2339.

Publish with Bio Med Central and every scientist can read your work free of charge

"BioMed Central will be the most significant development for disseminating the results of biomedical research in our lifetime."

Sir Paul Nurse, Cancer Research UK

Your research papers will be:

- available free of charge to the entire biomedical community

- peer reviewed and published immediately upon acceptance

- cited in PubMed and archived on PubMed Central

- yours - you keep the copyright

Submit your manuscript here:

http://www.biomedcentral.com/info/publishing_adv.asp
BioMedcentral 